\title{
A SNEVILY-TYPE INEQUALITY FOR MULTISETS
}

\author{
A. GÁSPÁR ${ }^{1}$ and G. KÓS $S^{2,3, *}$ \\ ${ }^{1}$ Mathematical Institute, Loránd Eötvös University, Pázmány Péter sétány 1/C, \\ H-1117 Budapest, Hungary \\ e-mail: gsprati99@gmail.com \\ ${ }^{2}$ Institute for Computer Science and Control (SZTAKI), P.O.Box 63, H-1518 Budapest, Hungary \\ e-mail: kosgeza@sztaki.hu \\ ${ }^{3}$ Alfréd Rényi Institute of Mathematics, P.O.Box 127, H-1364 Budapest, Hungary
}

(Received May 20, 2020; revised October 7, 2020; accepted October 9, 2020)

\begin{abstract}
Alon [1] proved that if $p$ is an odd prime, $1 \leq n<p$ and $a_{1}, \ldots$, $a_{n}$ are distinct elements in $Z_{p}$ and $b_{1}, \ldots, b_{n}$ are arbitrary elements in $Z_{p}$ then there exists a permutation of $\sigma$ of the indices $1, \ldots, n$ such that the elements $a_{1}+b_{\sigma(1)}, \ldots, a_{n}+b_{\sigma(n)}$ are distinct. In this paper we present a multiset variant of this result.
\end{abstract}

Motivation. Let $G$ be a finite group of odd order and suppose that $a_{1}, \ldots, a_{k} \in G$ are pairwise distinct and $b_{1}, \ldots, b_{k} \in G$ are pairwise distinct. Snevily's conjecture states that there is a permutation $\sigma$ of the indices $1,2, \ldots, n$ for which $a_{1} b_{\sigma(1)}, a_{2} b_{\sigma(2)}, \ldots, a_{k} b_{\sigma(k)}$ are pairwise distinct. The conjecture has been proved for cyclic groups of prime order by Alon, for cyclic groups by Dasgupta et al. [4] and for commutative groups by Arsovski [3].

Our motivation was to attack Snevily's conjecture in an inductive approach. Let $N$ be a maximal normal subgroup of $G$, so $p=G: N$ is an odd prime, for $|G|$ is odd and thus $\mathrm{G}$ is solvable. We look for a suitable matching of the cosets $a_{1} N, \ldots, a_{n} N$ and $b_{1} N, \ldots, b_{n} N$ first, to proceed among the elements in the cosets. Since we have $n>p$ in general, we cannot expect the cosets $a_{i} b_{\sigma(i)} N$ to be distinct. Instead we try to control the multiplicities in the sequence $\left(a_{1} b_{\sigma(1)} N, \ldots, a_{n} b_{\sigma(n)} N\right)$ and compare it with the multiplicities in $\left(a_{1} N, \ldots, a_{n} N\right)$ and $\left(b_{1} N, \ldots, b_{n} N\right)$. For such a program, we need a suitable multiset variant of Snevily's conjecture in the group $G / N \simeq Z_{p}$.

\footnotetext{
* Corresponding author.

Supported by National Research, Development and Innovation Office NKFIH Grant K 120154. Key words and phrases: Combinatorial Nullstellensatz, polynomial method, sumset, multiset, multiple point.

Mathematics Subject Classification: 05E40, 12D10.
} 
Notation. Throughout the paper, $p$ refers to an odd prime and $1 \leq n<p$ is an integer. $\operatorname{Sym}(n)$ denotes the set of permutations of $(1,2, \ldots, n)$. For $\sigma \in \operatorname{Sym}(n), \operatorname{sgn} \sigma$ denotes the sign of $\sigma$; that is, +1 for even permutations and -1 for odd permutations.

The boldface symbols denote sequences of $n$ objects, indiced by $1,2, \ldots, n$; in particular, $\mathbf{0}=(0, \ldots, 0)$ is the $n$-dimensional null vector. For any sequence $\mathbf{x}=\left(x_{1}, x_{2}, \ldots, x_{n}\right)$ and any permutation $\sigma \in \operatorname{Sym}(n)$, we define $\mathbf{x}^{\sigma}=\left(x_{\sigma(1)}, x_{\sigma(2)}, \ldots, x_{\sigma(n)}\right)$.

For any polynomial $P(\mathbf{x})$ with $n$ variables and nonnegatve integer vector $\mathbf{d}=\left(d_{1}, \ldots, d_{n}\right) \in \mathbb{N}^{n}, \partial^{\mathbf{d}} P(\mathbf{x})$ abbreviates the partial derivative $\partial x_{1}^{d_{1}} \ldots \partial x_{n}^{d_{n}} P\left(x_{1}, \ldots, x_{n}\right)$.

$V(\mathbf{x})=V\left(x_{1}, \ldots, x_{n}\right)=\prod_{1 \leq i<j \leq n}\left(x_{j}-x_{i}\right)$ is the Vandermonde polynomial with $n$ variables.

Results. We start with the following theorem of Alon:

TheOREM 1 (Alon [1]). Let $p$ be an odd prime, $1 \leq n<p$, and suppose that $a_{1}, \ldots, a_{n} \in \mathbb{F}_{p}$ are distinct and $b_{1}, \ldots, b_{n} \in \mathbb{F}_{p}$ arbitrary. Then there exists a permutation $\sigma$ of the indices $1,2, \ldots, n$ such that $a_{1}+b_{\sigma(1)}, \ldots$, $a_{n}+b_{\sigma(n)}$ are distinct.

Alon proved this theorem as an easy application of his powerful nonvanishing criterion (Theorem 1.2 in [2]), by examining the coefficient of $\left(x_{1} \cdots x_{n}\right)^{n-1}$ in the polynomial $V(\mathbf{x}) V(\mathbf{x}+\mathbf{b})$. Here we replicate a variant of the proof that can be extended to partial derivatives directly.

In order to state a multiset analogue, we define a quantity that measures the number of coinciding elements. For any finite sequence $\mathbf{x}=\left(x_{1}, \ldots, x_{n}\right)$, let $N(\mathbf{x})$ be the number of ordered index pairs $(i, j)$ with $1 \leq i<j \leq n$ and $x_{i}=x_{j}$. Notice that if there are $k$ different elements among $x_{1}, \ldots, x_{n}$ and they occur $m_{1}, \ldots, m_{k}$ times, respectively, then $N(\mathbf{x})=\sum\left(\begin{array}{c}m_{i} \\ 2\end{array}\right)$; if $x_{1}, \ldots$, $x_{n}$ are distinct, then $N(\mathbf{x})=0$. We will prove the following

Theorem 2. Let $p$ be an odd prime, $1 \leq n<p$, and let $\mathbf{a}, \mathbf{b} \in \mathbb{F}_{p}^{n}$. Then there exists a permutation $\sigma \in \operatorname{Sym}(n)$ such that

$$
N\left(\mathbf{a}+\mathbf{b}^{\sigma}\right) \leq N(\mathbf{a}) .
$$

Since $N\left(x^{\sigma}\right)=N(x)$, hence $N\left(a+b^{\sigma}\right)=N\left(b+a^{\sigma^{-1}}\right)$, an equivalent formulation is

$$
N\left(\mathbf{a}+\mathbf{b}^{\sigma}\right) \leq \min (N(\mathbf{a}), N(\mathbf{b})) .
$$

Alon's proof for Theorem 1 can be modified for this theorem; the necessary tools are presented in [5]. We prefer to give two independent proofs as below. 
Lemma 1. For any $\mathbf{b} \in \mathbb{F}_{p}^{n}$,

$$
\sum_{\sigma \in \operatorname{Sym}(n)} V\left(\mathbf{x}+\mathbf{b}^{\sigma}\right)=n ! \cdot V(\mathbf{x}) .
$$

Proof. Consider the polynomial

$$
P(\mathbf{x}, \mathbf{y})=\sum_{\sigma \in \operatorname{Sym}(n)} V\left(\mathbf{x}+\mathbf{y}^{\sigma}\right)=\sum_{\sigma \in \operatorname{Sym}(n)}(\operatorname{sgn} \sigma) \cdot V\left(\mathbf{x}^{\sigma^{-1}}+\mathbf{y}\right) .
$$

Given that $\operatorname{sgn}\left(\nu^{-1}\right)=\operatorname{sgn} \nu$ and $\operatorname{sgn}(\nu \tau)=\operatorname{sgn}(\nu) \operatorname{sgn}(\tau)$, this polynomial alternates in the variables in $\mathbf{x}$, so $P(\mathbf{x}, \mathbf{y})$ is divisible by $V(\mathbf{x})$. Since $P$ and $V$ have the same degree, $P(\mathbf{x}, \mathbf{y})$ must be some constant times $V(\mathbf{x})$; this constant can be determined by substituting $\mathbf{y}=\mathbf{0}$. Hence,

$$
\sum_{\sigma \in \operatorname{Sym}(n)} V\left(\mathbf{x}+\mathbf{b}^{\sigma}\right)=P(\mathbf{x}, \mathbf{b})=P(\mathbf{x}, \mathbf{0})=n ! \cdot V(\mathbf{x}) .
$$

Proof of Theorem 1. Substituting $\mathbf{x}=\mathbf{a}$ in (1) provides

$$
\sum_{\sigma \in \operatorname{Sym}(n)} V\left(\mathbf{a}+\mathbf{b}^{\sigma}\right)=n ! \cdot V(\mathbf{a}) \neq 0 .
$$

Therefore there is at least one nonzero term on the left-hand side, so there is a permutation $\sigma \in \operatorname{Sym}(n)$ such that $V\left(\mathbf{a}+\mathbf{b}^{\sigma}\right) \neq 0$, indicating that the elements in $\mathbf{a}+\mathbf{b}^{\sigma}$ are distinct.

Lemma 2. Let $\mathbf{a} \in \mathbb{F}_{p}^{n}$. Then

(a) For any $\mathbf{d} \in \mathbb{N}^{n}$ with $d_{1}+\cdots+d_{n}<N(\mathbf{a})$ we have $\partial^{\mathbf{d}} V(\mathbf{a})=0$.

(b) There exists $a \mathbf{d} \in \mathbb{N}^{n}$ such that $d_{1}+\cdots+d_{n}=N(\mathbf{a})$ and $\partial^{\mathbf{d}} V(\mathbf{a}) \neq 0$.

Proof. (a) Notice first that in $V(\mathbf{a})=\prod_{1 \leq i<j \leq n}\left(a_{j}-a_{i}\right)$ there are exactly $N(\mathbf{a})$ zero factors.

Suppose $d_{1}+\cdots+d_{n}=k<N(\mathbf{a})$. Notice that

$$
\partial^{\mathrm{d}} V(\mathbf{x})=\partial^{\mathrm{d}}\left(\prod_{1 \leq i<j \leq n}\left(x_{j}-x_{i}\right)\right)
$$

is a signed sum of subproducts of $\prod_{1 \leq i<j \leq n}\left(x_{j}-x_{i}\right)$, with each such product consisting of $\left(\begin{array}{l}n \\ 2\end{array}\right)-k$ factors. Substutiting $\mathbf{x}=\mathbf{a}$, each product contains at least $N(\mathbf{a})-k \geq 1$ zero factors.

(b) For $j=1, \ldots, n$, let $d_{j}$ be the number of indices $i$ with $1 \leq i<j$ and $a_{i}=a_{j}$. Then obviously $d_{1}+\cdots+d_{n}=N(\mathbf{a})$. Like in part (a), $\partial^{\mathbf{d}} V(\mathbf{x})$ is a is a signed sum of subproducts with $\left(\begin{array}{l}n \\ 2\end{array}\right)-N(\mathbf{a})$ factors. It can be seen that 
there is only one nonzero among them, which is the product of all nonzero factors, so with this choice of $\mathbf{d}$, we have $\partial^{\mathbf{d}} V(\mathbf{a}) \neq 0$ indeed.

First Proof For TheOrem 2. By part (b) of Lemma 2, there is some $\mathbf{d} \in \mathbb{N}^{n}$ such that $d_{1}+\cdots+d_{n}=N(\mathbf{a})$ and $\partial^{\mathbf{d}} V(\mathbf{a}) \neq 0$. Taking the $\mathbf{d}$-th partial derivative of (1),

$$
\sum_{\sigma \in \operatorname{Sym}(n)} \partial^{\mathbf{d}} V\left(\mathbf{a}+\mathbf{b}^{\sigma}\right)=n ! \cdot \partial^{\mathbf{d}} V(\mathbf{a}) \neq 0 .
$$

Hence, there is a $\sigma \in \operatorname{Sym}(n)$ such that $\partial^{\mathbf{d}} V\left(\mathbf{a}+\mathbf{b}^{\sigma}\right) \neq 0$; by part (a) of Lemma 2, we have

$$
N\left(\mathbf{a}+\mathbf{b}^{\sigma}\right) \leq d_{1}+\cdots+d_{n}=N(\mathbf{a})
$$

SECOND PROOF FOR ThEOREM 2. We prove by induction on $n$. The claim is trivial for $n=0$. Let $1 \leq n<p$, and assume that Theorem 2 is true for smaller values of $n$.

Let $k$ be the number of different elements among $a_{1}, a_{2}, \ldots, a_{n}$. Rearrange the elements in such an order that $a_{1}, a_{2}, \ldots, a_{k}$ are distinct.

Notice that each of $a_{k+1}, \ldots, a_{n}$ is listed exactly once among $a_{1}, a_{2}, \ldots$, $a_{k}$, so there are exactly $n-k$ pairs $i, j$ of indices with $i \leq k<j$ and $a_{i}=a_{j}$. Therefore,

$$
N\left(a_{1}, \ldots, a_{n}\right)=(n-k)+N\left(a_{k+1}, \ldots, a_{n}\right) .
$$

By Theorem 1 there is a permutation $\sigma_{1}$ of $1,2, \ldots, k$ such that $a_{1}+b_{\sigma_{1}(1)}, a_{2}+b_{\sigma_{1}(2)}, \ldots, a_{k}+b_{\sigma_{1}(k)}$ are distinct. By the induction hypothesis, there is a permutation $\sigma_{2}$ of $k+1, k+2, \ldots, n$ such that

$$
N\left(a_{k+1}+b_{\sigma_{2}(k+1)}, \ldots, a_{n}+b_{\sigma_{2}(n)}\right) \leq N\left(a_{k+1}, \ldots, a_{n}\right) .
$$

Merge $\sigma_{1}$ and $\sigma_{2}$ to a new permutation $\sigma$.

By the definition of $\sigma_{1}$, the elements $a_{1}+b_{\sigma(1)}, \ldots, a_{k}+b_{\sigma(k)}$ are distinct. For each $j$ with $k<j \leq n$, there is at most one index $i \leq k$ with $a_{i}+b_{\sigma(i)}=a_{j}+b_{\sigma(j)}$. For this reason,

$$
N\left(a_{1}+b_{\sigma(1)}, \ldots, a_{n}+b_{\sigma(n)}\right) \leq(n-k)+N\left(a_{k+1}+b_{\sigma(k+1)}, \ldots, a_{n}+b_{\sigma(n)}\right) .
$$

The estimates (2)-(4) together provide

$$
N\left(a_{1}+b_{\sigma(1)}, \ldots, a_{n}+b_{\sigma(n)}\right) \leq N\left(a_{1}, \ldots, a_{n}\right),
$$

completing the induction step.

At the end we remark that Theorems 1 and 2 are not true for $n=p$; an easy counter-example is $\mathbf{a}=(0,1,2, \ldots, p-1)$ and $\mathbf{b}=(1,0,0, \ldots, 0)$. 


\section{References}

[1] N. Alon, Additive Latin transversals, Israel J. Math., 117 (2000), 125-130.

[2] N. Alon, Combinatorial Nullstellensatz, Combin. Probab. Comput., 8 (1999), 7-29.

[3] B. Arsovski, A proof of Snevilys conjecture, Israel J. Math., 182 (2011), 505-508.

[4] S. Dasgupta, Gy. Károlyi, O. Serra and B. Szegedy, Transversals of additive Latin squares, Israel J. Math., 126 (2001), 17-28.

[5] G. Kós and L. Rónyai, Alon's Nullstellensatz for multisets, Combinatorica, 32 (2012), 589-605. 